\title{
Abdominal Tuberculosis Leading to Prehepatic Portal Hypertension: A Case Report
}

Xuelian Dan, Li Feng, Wanqin Wang and Li Yang

Division of Digestive Diseases, West China Hospital, Sichuan University, Chengdu 610041, PR China

*Corresponding author: Li Yang, Division of Digestive Diseases, West China Hospital, Sichuan University, Chengdu 610041, PR China, Tel/Fax: 86-28-85422311; Email: linayang63@gmail.com

Rec date: Aug 02, 2014, Acc date: Sep 09, 2014, Pub date: Sep 09, 2014

Copyright: (c) Dan X, et al. This is an open-access article distributed under the terms of the Creative Commons Attribution License, which permits unrestricted use, distribution, and reproduction in any medium, provided the original author and source are credited.

\begin{abstract}
Tuberculosis can affect any organ or tissue in the abdomen such as gastrointestinal tract, peritoneum, lymphatic system and solid organs. Lymphadenopathy is the most common manifestation of abdominal tuberculosis which can be the only sign of the disease, especially in the periportal region or combined with peritoneal or solid organ involvement. There are a number of causes for prehepatic noncirrhotic portal hypertension, but there were a few reports of prehepatic portal hypertension associated with tuberculosis. Here we report a rare case of intra-abdominal tuberculosis as a cause for portal hypertension and recurrent gastrointestinal bleeding. Enlarged lymph nodes at hepatic hilum compressed the portal vein which caused portal hypertension and portal hypertensive gastropathy and enteropathy. The patient was managed by anti-tuberculosis therapy followed by splenectomy, surgical portaazygous devascularization and splenorenal shunt. In the 2-year followup after operation, the patient was free of symptoms and had cavernous portal vein transformation on CT, and disappearance of varices and portal hypertensive gastroenteropathy on EGDs.
\end{abstract}

Keywords: Abdominal tuberculosis; Prehepatic portal hypertension; Portal hypertensive gastropathy and Enteropathy

\section{Introduction}

Tuberculosis (TB) remains a worldwide health problem and the incidence has increased in the developed countries owing to immigration and the AIDS epidemic. The infection modes include haematogenous spread from active tuberculosis, ingestion of infected sputum or milk and direct extension from adjacent organs. Abdominal tuberculosis is a common extrapulmonary tuberculosis site, which accounts for $4.9 \%$ of all cases of tuberculosis. It can involve intestinal tract, peritoneum, solid organs (liver, spleen), lymphatic system as well as genitourinary tract [1-3]. Tuberculosis lymphadenopathy is the most common intraabdominal manifestation, which commonly involves peripancreatic, portal hepatis, mesenteric and omental lymph nodes $[3,4]$.

Portal hypertension $(\mathrm{PH})$ is a common clinical syndrome that is most often caused by liver cirrhosis. $\mathrm{PH}$ can also be caused by many noncirrhotic etiologies. Based on the site of portal flow obstruction, it is classified as pre-hepatic, hepatic, post-hepatic. Prehepatic portal hypertension $(\mathrm{PPH})$ can be associated with any disease that causes the obstruction of portal vein, such as portal vein thrombosis [5]. Gastrointestinal bleeding is a life-threatening complication of $\mathrm{PH}$ and is most commonly caused by esophageal varices or gastric varices. Other causes for bleeding include portal hypertensive gastropathy, enteropathy, gastric antral vascular ectasia (GAVE), etc. Treatment for GI bleeding from portal hypertensive gastroenteropathy is to reduce portal hypertension [6]. Here we report a case of intraabdominal tubercular lymphadenopathy leading to $\mathrm{PPH}$, portal hypertensive gastroenteropathy and gastrointestinal bleeding.

\section{Case Report}

A male patient, aged 33, was hospitalized on April 28, 2012 due to "repeated melena 1-2 times every day, fatigue, dizziness and exertional dyspnea for two months". 9 months prior to this presentation, the patient was admitted to oncology department of our hospital because of abdominal pain, distension and abdominal mass. Then he denied a medical history of hepatitis, tuberculosis, cirrhosis of the liver or pancreatitis and he had a history of smoking for 10 years, 20 cigarettes every day. Physical examination revealed enlarged several cervical and axillary lymph nodes. He was suspected of having an abdominal malignancy. An abdominal contrast-enhanced computed tomography (Figure 1) showed soft-tissue masses at the hepatic hilum, an enlargement of celiac and retroperitoneal lymph nodes, edematous omentum and mesenterium as well as ascites. There was no radiographic evidence of portal hypertension at that time, such as varices. An esophagogastroduodenoscopy (EGD) indicated chronic superficial gastritis without varices and a colonoscopy was normal. Laparoscopic biopsy was performed and histopathological examination revealed granuloma with necrosis (Figure 2). Polymerase chain reaction (PCR) of the biopsy was positive for TB. No malignancy was found. He was put on oral antitubercular therapy (rifampicin, isonaizid, ethambutol, ofloxacin) since then.

Upon physical examination on the current presentation, his vital signs were normal and he weighed 56 kilograms. Several spider nevi on the chest, skin pallor and a markedly enlarged spleen $(4 \mathrm{~cm}$ below left costal margin) were noted. The abdomen was soft without palpable masses. Routine laboratory findings were unremarkable except for a hemoglobin level of $69 \mathrm{~g} / \mathrm{L}$. The serologic tests for viral hepatitis, HIV, syphilis and autoimmune antibodies were negative. An abdominal contrast-enhanced computed tomography (Figure $3 \mathrm{~A}$ and $3 \mathrm{~B}$ ) revealed enlarged lymph nodes compressing on the portal vein at the portacaval space and above the head of pancreas, splenomegaly, 
splenic varices, left gastric varices and esophagogastric varices. Magnetic resonance imaging (Figure 3C) had similar findings but also revealed a dilation of portal vein and biliary ducts. The liver was normal on both CT and MRI. An EGD (Figure 4) showed portal hypertensive gastroduodenopathy (A) and mild esophageal varices (B). A capsule endoscopy (CE) (Figure 4C and 4D) found vascular tortuosity and mild errhysis in the jejunum.

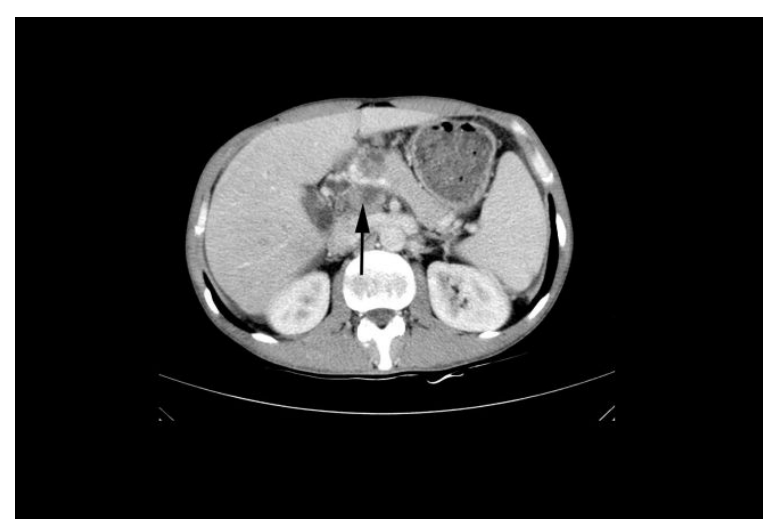

Figure 1: Abdominal contrast-enhanced computed tomography images. Soft-tissue mass with nonhomogeneous density was located in the periportal area (arrow) and it could not be separated from the pancreas.

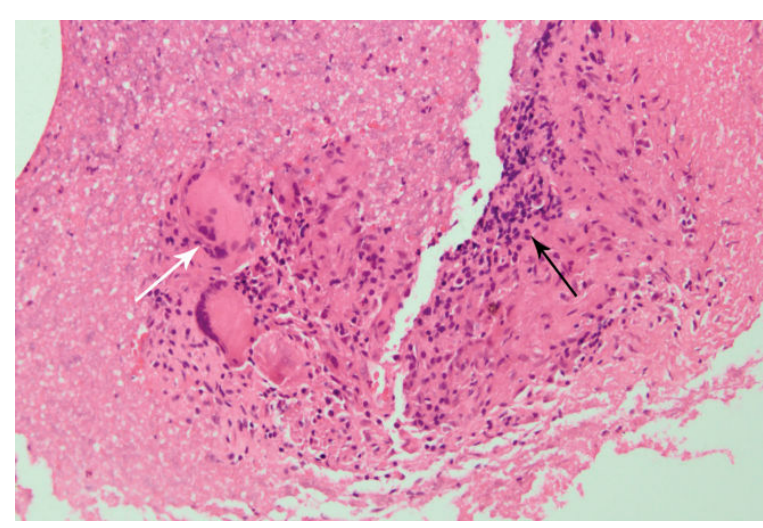

Figure 2: Histopathological examination of abdominal mass. There were several Langhans' giant cells (white arrow) besides granulomatous inflammation with fibrosis (black arrow) (H\&E, $\mathrm{x} 200)$.

Despite medical and endoscopic management, the patient continued to have melena. Transjugular intrahepatic portosystemic shunt (TIPS) was not expected to relieve the portal hypertension in this patient with prehepatic portal vein obstruction. A surgical consultation was obtained and the patient underwent splenectomy, porta-azygous devascularization and splenorenal shunt on May 25, 2012. Intra-operative findings included an enlargement of spleen $(14 \times 10 \times 8 \mathrm{~cm})$, thickened porta hepatis and varices in the lower esophagus and fundus of stomach. Histopathologic examination of spleen and cardiac left lymph nodes revealed spleen congestion and caseation necrosis with fibrous tissue respectively. The patient was discharged eight days after the operation and completed a total of 18month tuberculosis therapy. He was followed up for about two years. He took antitubercular agent regularly and came to have blood tests and imaging tests at regular intervals. He did well without any bleeding or other symptoms. He had two EGDs (Figure 5) since the operation which showed no varices or portal hypertensive gastroenteropathy. An abdominal contrast-enhanced computed tomography (Figure 3D) two years after operation indicated cavernous transformation of portal vein with no varices and enlarged lymph nodes. And the patient was asked to have regular reexamination every 3 months and come to hospital if there is any discomfort.

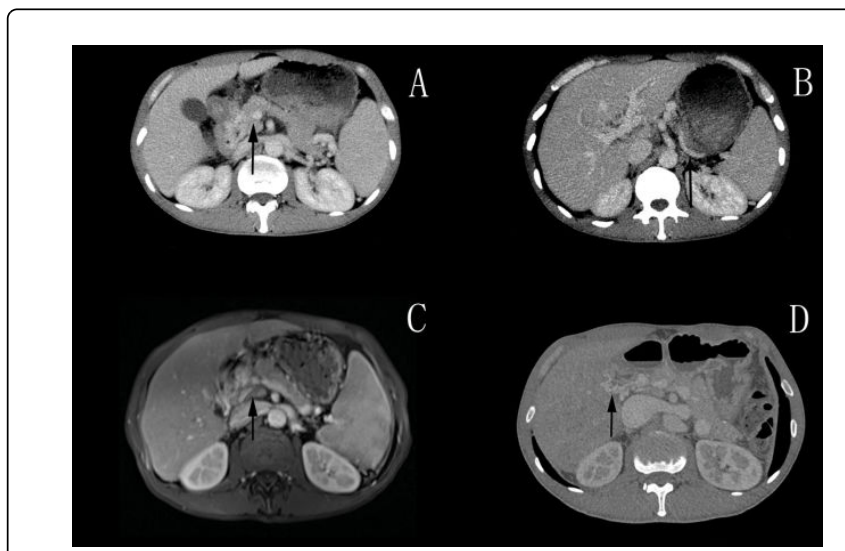

Figure 3: Abdominal CT images. (A, B) Enlarged lymph nodes (arrow) compress the portal vein and varices appeared (arrow). (C) Magnetic resonance images. Enlarged lymph nodes (arrow) above the pancreas oppress the portal vein. (D) Cavernous transformation (arrow) of portal vein appeared and there were no varices or enlarged lymph nodes two years after the operation.

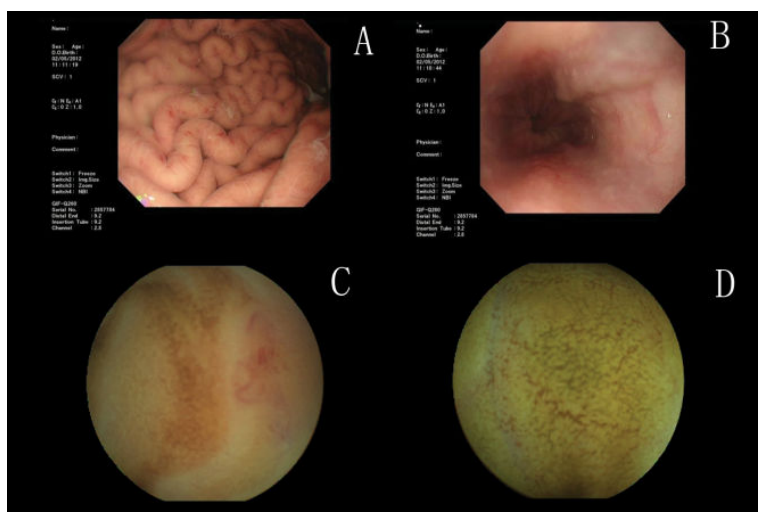

Figure 4: Gastroduodenal appearance in endoscopy. (A) We could find some manifestinations of portal hypertensive gastropathy such as mucosal edema and red spots. (B) Mild esophageal varices (B) was also observed. (C, D) A capsule endoscopy. There was tortuosity of submucosa vessel and small amount of errhysis. 


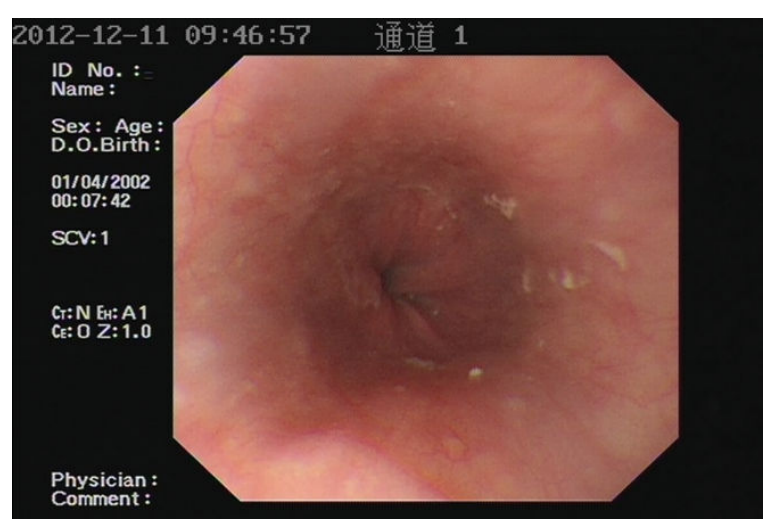

Figure 5: Upper gastrointestinal endoscopy. Gastroesophageal varices disappeared after shunt operation.

\section{Discussion}

Abdominal tuberculosis is a common location for extra-pulmonary tuberculosis. The classic symptoms of pulmonary $\mathrm{TB}$ such as intermittent fever, weight loss and night sweats are sometimes absent $[1,7]$. Common clinical manifestations of abdominal TB such as abdominal pain, distension, masses and ascites are similar to other intra-abdominal diseases (abdominal malignancy, Crohn's disease, etc.). Moreover, routine laboratory and radiographic studies are often non-specific, thus abdominal $\mathrm{TB}$ remains a diagnostic challenge in clinical practices $[1,8,9]$. At present, a diagnostic laparoscopy is often required for the diagnosis of abdominal TB.

Portal hypertension is a common complication of cirrhosis which is defined as a portal vein pressure increased above the upper normal limit by $10 \mathrm{mmHg}$, but it can also occur in non- cirrhotic patients $[5,10]$. The cause of prehepatic portal hypertension varies with the patient [11]. Any lesions leading to the obstruction or increased blood flow of portal vein or splenic vein can result in PPH. Common lesions include abdominal neoplasms compressing the portal or splenic veins, inflammation lesions (umbilical infection, appendicitis, sepsis, biliary tract disease, etc.), direct or indirect portal or splenic vein injury (trauma, operation, etc.), pancreatitis, splenic artery aneurysm, etc. Celiac and retroperitoneal tuberculosis nodules oppressing the portal vein, congenital portal-caval fistula, splanchnic arteriovenous fistula, congenital portal venous malformations and hypercoagulable state are some other uncommon factors. Moreover, some hematological diseases including Lymphoma, myeloproliferative disorders and Gaucher's disease can lead to massive splenomagly which cause increased blood flow in splenic vein thus leading to the formation of PPH $[5,11]$.

Nine months prior to his gastrointestinal bleeding, the patient was admitted to oncology with a history of abdominal pain, distension and mass. EGD and colonoscopy showed no obvious lesions. Abdominal CT (Figure 1) indicated abdominal mass, enlarged lymph nodes as well as ascites. These are common manifestations of intra-abdominal malignancy. The final diagnosis of abdominal tuberculosis was not established until after laparoscopic intra-abdominal lymph node biopsies showing caseating granuloma and a positive tuberculosis PCR test using the biopsy tissue. Malignancy and abdominal tuberculosis can mimic each other which often lead to misdiagnosis. Laparoscopic biopsy proves to be the most valuable in differentiating abdominal tuberculosis and malignancy.

The patient's portal hypertension was not evident on initial presentation and his gastrointestinal bleeding did not occur until after 9 months of tuberculosis treatment. The abdominal CT and MRI both showed lymphadenopathy and portal vein obstruction despite 9 months of tuberculosis treatment. Medical treatment of tuberculosis alone is apparently not adequate to reverse the progression of portal hypertension in this patient. We found no viral hepatitis or other liver diseases in this patient. There was no evidence of hepatic tuberculosis on the laparoscopic exploration. Imaging tests revealed no posthepatic lesions. Thus this is a case of prehepatic portal hypertension caused by abdominal tuberculosis. The possible mechanisms for development of portal hypertension may include enlarged lymph nodes compressing on portal vein and/or inflammation and tissue fibrosis constricting the portal vein. Abdominal tuberculosis is rare, but it needs to be included in the differential diagnosis of prehepatic portal hypertension in the right clinical setting.

The clinical features of prehepatic portal hypertension are similar to portal hypertension in cirrhosis and other portal hypertensive diseases. Common symptoms include upper gastrointestinal bleeding, splenomegaly, hypersplenism and ascites [5]. Though variceal hemorrhage is the leading cause for gastrointestinal bleeding in portal hypertension, portal hypertensive gastropathy and enteropathy represent important causes of chronic gastrointestinal bleeding. It can involve the stomach, duodenum, jejunum, and distal ileum. It is defined as mucosal abnormalities in gastrointestinal tract without inflammatory change on histologic examination. While portal hypertensive gastropathy is easily identified by EGD, capsule endoscopy is needed for the diagnosis of portal hypertensive enteropathy. The characteristic lesions on endoscopy include diffuse mucosal edema and erosion, red spots, vascular tortuosity and increasing number of vessel and angiectasia [12-14]. Management of bleeding from portal hypertensive gastroenteropathy can be challenging often because of diffuse bleeding sites. Vasoconstrictors such as terlipressin, somatostain and its analogues may be used but not very effective. Transjugular intrahepatic portosystemic shunt (TIPS) can be very effective for treating intra-hepatic and some post-hepatic portal hypertension bleeding. But TIPS is not useful for prehepatic portal hypertension. Thus sugery remains an important option for these patients. Successful surgery can relieve obstruction of portal vein and reduce portal hypertension. Shunt surgery and ablative procedures can be applied individualized to patients according to patient's different conditions [15]. In our case, EGD and CE indicated bleeding portal hypertensive gastropathy and enteropathy. Vasoconstrictors combined with endoscopic therapy were not effective in controlling the GI bleeding. So the patient underwent splenectomy, esophagogastric devascularization and splenorenal shunt. In the 2-year following-up, the patient did very well without any signs of bleeding and he had a complete resolution of esophageal varices and portal hypertensive gastroenteropathy .

In conclusion, abdominal tuberculosis is a rare cause of prehepatic hypertension and medical treatment for TB alone may not reverse the progression of portal hypertension and the risk of GI bleeding. Medical and endoscopic management of GI bleeding associated with prehepatic portal hypertension is difficult and often not effective. Thus surgical intervention including splenectomy, porta-azygous devascularization and splenorenal shunt may be needed. 
Citation: Dan X, Feng L, Wang W, Yang L (2014) Abdominal Tuberculosis Leading to Prehepatic Portal Hypertension: A Case Report. J Cytol Histol 5: 278. doi:10.4172/2157-7099.1000278

Page 4 of 4

\section{References}

1. Burke KA, Patel A, Jayaratnam A, Thiruppathy K, Snooks SJ (2014) Diagnosing abdominal tuberculosis in the acute abdomen. Int J Surg 12: 494-499.

2. Ozseker B, Ozseker HS, Kav T, Shorbagi A, Karakoc D, et al. (2012) Abdominal tuberculosis leading to portal vein thrombosis, mimicking peritoneal carcinomatosis and liver cirrhosis. Acta Clin Belg 67: 137-139.

3. Pereira JM, Madureira AJ, Vieira A, Ramos I (2005) Abdominal tuberculosis: imaging features. Eur J Radiol 55: 173-180.

4. Lee WK, Van Tonder F, Tartaglia CJ, Dagia C, Cazzato RL, et al. (2012) CT appearances of abdominal tuberculosis. Clin Radiol 67: 596-604.

5. Khanna R, Sarin SK (2014) Non-cirrhotic portal hypertension - diagnosis and management. J Hepatol 60: 421-441.

6. Kalafateli M, Triantos CK, Nikolopoulou V, Burroughs A (2012) Nonvariceal gastrointestinal bleeding in patients with liver cirrhosis: a review. Dig Dis Sci 57: 2743-2754.

7. Singal R, Mittal A (2013) Plasted abdominal tuberculosis presenting as an obstruction. J Infect Dev Ctries 7: 561-563.

8. Tarcoveanu E, Dimofte G, Bradea C, Lupascu C, Moldovanu R, et al (2009) Peritoneal tuberculosis in laparoscopic era. Acta Chir Belg 109: 65-70.
9. Song J, Li H, Li Y, Yu H, Li X, et al. (2014) Is PET-CT scan an effective tool to differentiate the ascites caused by abdominal malignancy and peritoneal tuberculosis? Clin Res Hepatol Gastroenterol 38: e41-43.

10. Strauss E, Valla D (2014) Non-cirrhotic portal hypertension - Concept, diagnosis and clinical management. Clin Res Hepatol Gastroenterol.

11. Okuda K (2002) Non-cirrhotic portal hypertension versus idiopathic portal hypertension. J Gastroenterol Hepatol 17 Suppl 3: S204-213.

12. Dabos KJ Koulaouzidis A (2014) Portal hypertensive enteropathy, occult bleeding, and capsule endoscopy: where do we go from here? Dig Dis Sci 59: 899-901.

13. Patwardhan VR, Cardenas A (2014) Review article: the management of portal hypertensive gastropathy and gastric antral vascular ectasia in cirrhosis. Aliment Pharmacol Ther 40: 354-362.

14. De Palma GD, Rega M, Masone S, Persico F, Siciliano S, et al. (2005) Mucosal abnormalities of the small bowel in patients with cirrhosis and portal hypertension: a capsule endoscopy study. Gastrointest Endosc 62: 529-534.

15. Gauthier F (2005) Recent concepts regarding extra-hepatic portal hypertension. Semin Pediatr Surg 14: 216-225. 